\title{
Digitalisierungsprojekte professionell managen
}

\author{
Gabriele Korge, Alexandra Mantsch, Anja Serra \\ und Agnes Bauer
}

\begin{tabular}{l|l}
\multicolumn{1}{l}{ Steckbrief } & \multicolumn{2}{l}{$\begin{array}{l}\text { MARVECS GmbH, Ulm } \\
\text { MARVECS ist mit über 600 Mitarbeitern einer der großen Anbieter von } \\
\text { Außendienstlösungen und Vertriebsspezialist für die Healthcare-Branche. }\end{array}$} \\
\hline Auftrag & $\begin{array}{l}\text { Lernziel war, im Leitungsteam Know-how zu Change Management und zur } \\
\text { beteiligungsorientierten Einführung neuer Software aufzubauen. } \\
\text { Projektziel war, ein Social Intranet als interne, zentrale Kommunikations- und } \\
\text { Austauschplattform aufzusetzen. }\end{array}$ \\
\hline Rollen & $\begin{array}{l}\text { Lernende: Die Leiterin Finance \& Human Resources und ein IT Operations } \\
\text { Manager } \\
\text { Begleiterinnen: Eine Mitarbeiterin von MARVECS als methodische } \\
\text { Begleiterin, eine externe Expertin für die fachliche Begleitung } \\
\text { Auftraggeberin: Die Geschäftsführerin }\end{array}$ \\
\hline Ablauf & $\begin{array}{l}\text { In vier Monaten wurden vier Etappen mit einer Länge von jeweils 3 bis } \\
\text { 8 Wochen durchgeführt. Der Lernaufwand betrug ca. eine Stunde pro Woche. }\end{array}$ \\
\hline Technik & $\begin{array}{l}\text { „Microsoft Teams“, das bereits für die interne Projekt- und Zusammenarbeit, } \\
\text { die Arbeitsplanung und die direkte Kommunikation genutzt wird, wurde auch } \\
\text { für das Intranet- und das Lernprojekt genutzt. }\end{array}$ \\
\hline
\end{tabular}

G. Korge $(\bowtie)$

Fraunhofer-Institut für Arbeitswirtschaft und Organisation IAO, Stuttgart, Deutschland

E-Mail: gabriele.korge@iao.fraunhofer.de

A. Mantsch · A. Serra

Marvecs GmbH, Ulm, Deutschland

E-Mail: alexandra.mantsch@marvecs.de; anja.serra@marvecs.de

A. Bauer

ZNL TransferZentrum für Neurowissenschaften und Lernen der Universität Ulm, Ulm, Deutschland

E-Mail: agnesbauer@web.de 


\begin{tabular}{l|l}
\hline Besonderheiten & $\begin{array}{l}\text { Die Entscheidung für das Lernprojekt fiel einige Wochen nach Start des } \\
\text { Intranet-Projektes und nur wenige Monate vor dem geplanten Release des } \\
\text { Intranets. Die Vorbereitung zum agilen Lernen erfolgte folglich unter großem } \\
\text { Zeitdruck. } \\
\text { Zudem stand der Aufbau von grundlegendem Change Management Know-how } \\
\text { immer unter dem Vorbehalt der unmittelbaren Nützlichkeit für das Intranet- } \\
\text { Projekt. }\end{array}$ \\
\hline
\end{tabular}

\subsection{Anlass und Ausgangssituation}

\section{Mitarbeiterbindung als Herausforderung}

MARVECS ist mit über 600 Mitarbeitern einer der großen Anbieter von Außendienstlösungen und Vertriebsspezialist für die Healthcare-Branche. Über 40 Kunden bietet MARVECS deutschlandweit die vollständige Palette aller Maßnahmen, die für die erfolgreiche Einführung und Vermarktung von Healthcare-Produkten erforderlich ist. Zum Leistungsspektrum zählen die Realisierung von innovativen Vertriebswegen sowie die gezielte Rekrutierung von Vertriebssprofis für die Zielgruppe Krankenhäuser, Ärzte und Apotheker. Getreu dem Motto ,sMARt in die Zukunft - Wir setzen auf agile Methoden und realisieren Vertriebserfolge für unsere Kunden“ setzt MARVECS dabei auf innovative Arbeitsmethoden.

Da der persönliche Kontakt zu den Außendienstmitarbeitern nur selten möglich ist, stellt die Mitarbeiterbindung eine besondere Herausforderung dar. Um die Unternehmenskommunikation und die Identifikation mit MARVECS zu stärken, sollte ein Social Intranet aufgesetzt werden. Ziel der Geschäftsführerin war, die Kommunikation zu erleichtern und dafür eine zentrale Plattform bereitzustellen.

Von dieser aus sollten alle weiteren, die Arbeit unterstützenden digitalen Anwendungen erreicht sowie Neuigkeiten und Informationen abgerufen werden können. Zukünftige Nutzer dieser Plattform sollten im ersten Schritt der Innendienst am Hauptstandort in Ulm sowie die deutschlandweit verteilten Projekt- und Regionalleiter sein. Insbesondere der Austausch zwischen dem Innendienst und den Projekt- und Regionalleitern sollte auf diesem Weg unterstützt werden. Jede und jeder sollte sich schnell und einfach über die neusten Geschehnisse informieren und auch eigene Neuigkeiten mitteilen können.

\section{Das Intranet-Projekt}

Zum Start des Intranet-Projektes gab es bei MARVECS bereits erste Erfahrungen in der agilen Einführung neuer Software unter Beteiligung der Betroffenen. Die Vorteile - hohe Akzeptanz, Machbarkeit trotz hoher Arbeitsbelastung, schnelle erste Erfolge - führten dazu, dass auch das Intranet-Projekt als agiles Change-Vorhaben aufgesetzt wurde.

Die Projektleitung übernahm die Leiterin Finance \& Human Resources. Sie stellte ein abteilungsübergreifendes Projektteam zusammen, dem auch ein IT Operations Manager angehörte. Die Projektaufgaben organisierte sie ähnlich wie in einem Scrum-Projekt mit 
zweiwöchigen Sprints. Alle zwei Wochen traf sie sich mit dem Projektteam, um den Fortschritt der Arbeiten zum Intranet sowie die neu anstehenden Arbeitsaufgaben zu besprechen und zu planen. Eine Kollegin, die bereits etwas Übung in der methodischen Begleitung solcher Projekte hatte, unterstützte die Projektleiterin organisatorisch und in der Moderation.

Das Intranet-Projekt hatte bereits mehrere Zwei-Wochen-Schleifen durchlaufen, als die Projektleiterin trotz erfolgreich erarbeiteter Zwischenergebnisse immer mehr den Eindruck gewann, dass das Vorhaben nicht dynamisch, sondern zu starr verlaufen würde. Grund dafür war u. a. das fehlende Wissen zur Umsetzung eines Change-Projekts mit agilen Methoden.

So kam der Projektleiterin der Gedanke, ein agiles Lernprojekt aufzusetzen: Von den Kollegen kannte sie die Erfolgsstory eines früheren agilen Lern- und Changeprojektes zur Einführung einer neuen Software. Nun wollte sie, parallel zum Intranet-Projekt, lernen, wie ein agiles Change-Vorhaben zu steuern und zu managen ist. Die Geschäftsführerin unterstützte die Pläne der Projektleiterin, ihr war aber auch wichtig, dass sich das IntranetProjekt dadurch nicht verzögert.

\subsection{Zehn Tage zur Vorbereitung des Lernprojekts}

Zur Vorbereitung des Lernprojektes wurde kurzfristig ein zweistündiges Arbeitstreffen aufgesetzt; mehr Zeit fand sich nicht in den Kalendern der Projektleiterin, ihrer Kollegin, die das Intranet-Projekt methodisch begleitete und einer externen Expertin für Organisationsentwicklung und agiles Lernen. An diesem Termin wurden Lernziel und -thema geklärt, die Rollen besetzt und der Ablauf des agilen Lernens organisiert.

\section{Auftragsklärung: Ein Lernprojekt, zwei Ziele}

Zwei Lernziele wurden in Abstimmung mit der Projektleiterin vereinbart:

- Sie wollte, parallel zum Fortschritt des Intranet-Projektes, jeweils das an agilen ChangeMethoden und Vorgehensweisen lernen, was sie unmittelbar für den nächsten Sprint im Intranet-Projekt benötigte.

- Darüber hinaus wollte sie aber auch grundlegende Kompetenzen zur Steuerung und zum Management von agil aufgesetzten Change Vorhaben erwerben. Dies war auch der Geschäftsführerin wichtig, denn bei MARVECS standen weitere Projekte zur Reorganisation und zur Digitalisierung an.

\section{Rollenklärung: Erweitertes Steuerungs- und Lernteam}

Zum Lernteam sollte neben der Projektleiterin auch der IT Operations Manager gehören, der aktuell noch Teil des Intranet-Projektteams war: Die externe Expertin teilte die Einschätzung der Projektleiterin, dass er dort faktisch ohnehin bereits Management- und 
Steuerungsverantwortung trug und besser mit der Projektleiterin ein Team zur Steuerung des Intranet-Projektes bilden sollte. Da er sicher auch in zukünftigen Projekten in dieser Rolle gebraucht würde, war er auch bereit, sich die dazu benötigten agilen ChangeMethoden anzueignen. Die Leiterin Finance \& Human Resources und der IT Operations Manager bildeten in der Folge ein Steuerungs- und Lernteam (s. Abb. 9.1).

Die methodische Begleiterin des Intranet-Projekts sollte diese Rolle auch im Lernprojekt übernehmen: Sie hatte bereits Erfahrung mit der Begleitung agiler Lernprojekte und auch Schulungen dazu erhalten, wie sich Lernende optimal unterstützen und aktivieren lassen (für mehr Informationen zur Qualifizierung, s. Kap. 13).

Die fachliche Begleitung sollte durch die externe Expertin erfolgen, zumal zur Begleitung dieses Themas weniger internes Wissen als vielmehr Projekt-Erfahrung im agilen Change Management benötigt wurde.

\section{Lernorganisation: Bestimmt durch das zeitlich Machbare}

Vorgesehen war zunächst, die Lernorganisation auf das Intranet-Projekt abzustimmen, mit zweiwöchigen Etappen und Etappenmeetings am Tag der Intranet-Projektmeetings, evtl. auch einen Tag früher. Das ließ sich jedoch nicht mit den Terminkalendern aller Beteiligten vereinbaren. So ergab sich ein eher unrhythmischer Ablauf mit vier Etappen von drei, vier, drei und acht Wochen (s. Abb. 9.1). Die Etappentreffen wurden auf je 1,5 Stunden angesetzt. Dies sollte ausreichen, wie die Erfahrung aus anderen Lernprojekten, auch bei MARVECS, gezeigt hatte.

\section{Lernaufgaben: Auf Vorrat aufgesetzt}

Auch die inhaltliche Vorbereitung erfolgte aufgrund des Zeitdrucks auf eher unübliche Weise. Ein ideales Vorgehen wäre gewesen, zunächst umfassende Analysen anzustellen: Welches Vorwissen bringen die Lernenden mit? Was ist der Stand des Intranet-Projektes? Welches Wissen würde aktuell und in zukünftigen Change Vorhaben benötigt? Welche Kompetenzlücken ergeben sich daraus? Ausgehend davon würden dann Lernthemen bestimmt und Lernaufgaben formuliert.

Stattdessen fragte die externe fachliche Begleiterin lediglich grob das vorhandene Vorwissen zu (Lean) Change Management und zu allgemeinem Projektmanagement sowie den Stand des Intranet-Projekts ab. Ausgehend davon erstellte sie, quasi auf Vorrat, eine umfassendere Sammlung an Lernaufgaben zu typischen Herausforderungen, wie sie sich früher oder später in (Lean) Change Vorhaben stellen. Aus diesen sollte das Lernteam dann in der Planung zu den Etappen die Themen auswählen, die sich mit aktuellen Projektherausforderungen decken bzw. helfen, Versäumtes nachzuholen. 
Doppelte Rolle des Steuerungsund Lernteams

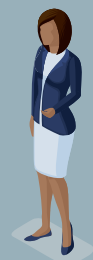

Leiterin Finance \& Human Resources

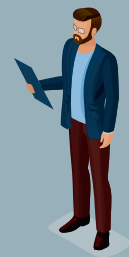

IT Operations Manager
Steuerungsteam für Intranet-Projekt
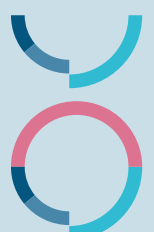

je 2

Wochen
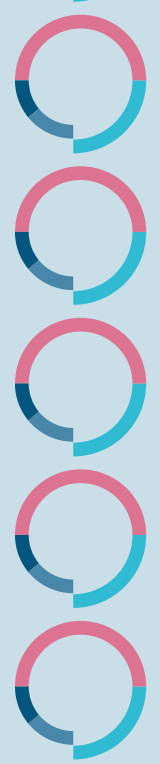

Release Intranet
Lernteam zum Thema agiles

Change Management

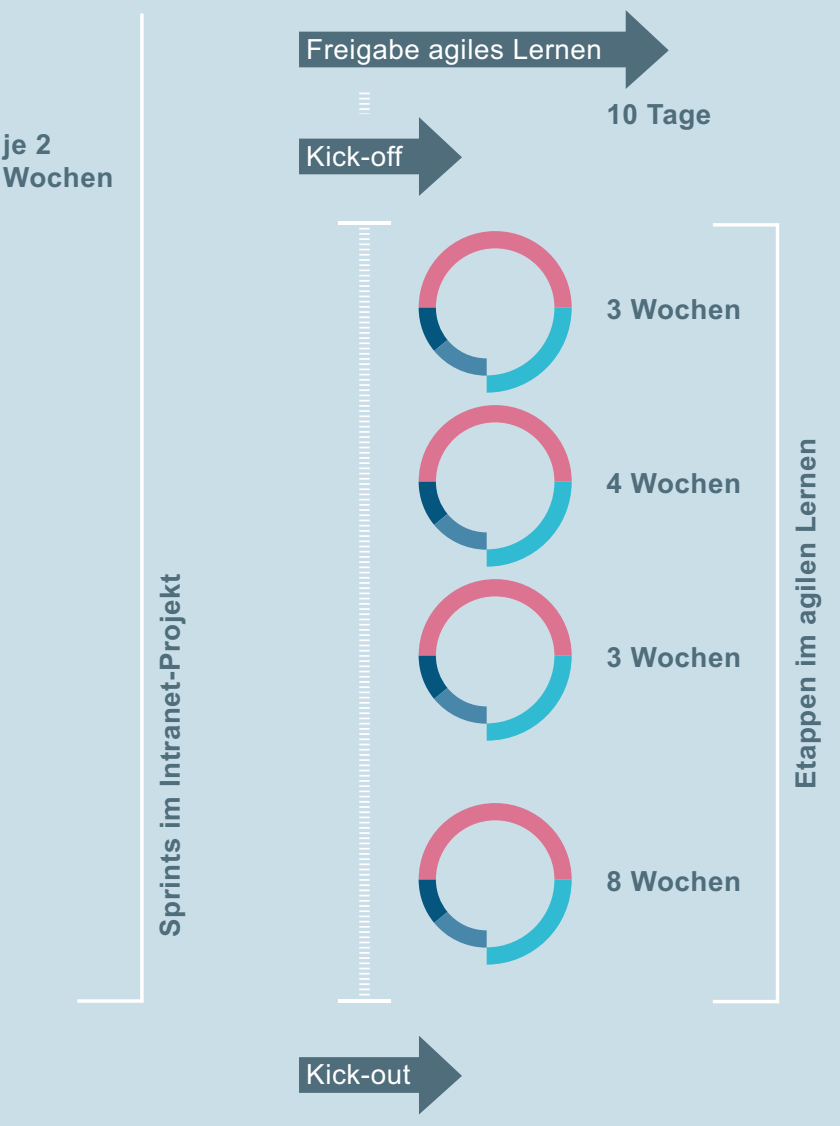

Abb. 9.1 Rollen und Organisation von Intranet- und Lernprojekt 


\subsection{Los geht's: Lernen auf der Überholspur}

Zum Auftakt des Lernens trafen sich die Lernenden mit der methodischen und der fachlichen Begleiterin zu einem 1,5-stündigen Kick-off. Hier informierte zunächst die methodische Begleiterin die Lernenden über den Ablauf und stimmte sie in ihre Rollen ein. Dann umriss die fachliche Begleiterin das Lernthema. Sie erläuterte, welche Teilaufgaben typischerweise anstehen, welche besonderen Herausforderungen das Change Management mit sich bringt und woran solche Projekte typischerweise scheitern.

Die Lernenden sollten selbst erkennen können, wo sie im Intranet-Projekt stehen und auf welchen nächsten Schritt sie sich besonders vorbereiten sollten. Die anschließende offene Diskussion zeigte dann zum Beispiel, dass die Projektleiterin und der IT Operations Manager zwar jeder für sich eine klare Vorstellung vom zu erreichenden Ergebnis „Intranet" hatten, es aber kein gemeinsames, formuliertes Zielbild gab. In der Folge fand keine Abstimmung dieses Zielbilds mit der Geschäftsführerin statt und auch dem IntranetProjektteam fehlte dadurch ein festgelegtes Zielbild zur Orientierung.

Ausgehend von diesem klareren Bild zum Stand des Intranet-Projektes und zu anstehenden Herausforderungen wählten die Lernenden in Abstimmung mit der fachlichen Begleiterin in der ersten Planungsrunde zwei Lernaufgaben aus: die Formulierung einer Strategie sowie die Entwicklung eines Zielbilds (Abb. 9.2 zeigt exemplarisch die Lernaufgabe zum Zielbild). In den folgenden Etappen bearbeiteten die Lernenden auch Aufgaben zur Entwicklung einer Roadmap, zum Finden der richtigen Optionen, zum Bestimmen eines Beteiligungsmodells, zum Entwerfen eines Kommunikationskonzepts und zum Erzeugen von Quick Wins - ersten Erfolgen also, die in kürzester Zeit erreichbar waren.

In der nun folgenden Etappe bearbeiteten die Lernenden die eingeplanten Aufgaben selbstständig, während die methodische Begleiterin den Fortschritt verfolgte. Dies konnte sie bei eher zufälligen Begegnungen und durch Rückfragen tun, da alle am gleichen Standort arbeiteten. Sie nutzte aber auch das Planungstool MS Teams. In dem dort für das Lernprojekt angelegten Kanban-Board war der Lernfortschritt jederzeit erkennbar (s. Abb. 9.3).

Die Meetings zum Etappenwechsel starteten jeweils mit dem Review, in dem die Lernenden der fachlichen Begleiterin ihre Ergebnisse präsentierten. Dieser Teil des Etappenwechsels nahm mit bis zu einer Stunde Dauer die meiste Zeit in Anspruch: Neben der Präsentation der Ergebnisse erfolgte ein intensiver Austausch mit der fachlichen Begleiterin, die von konkreten Change Vorhaben berichtete und es so den Lernenden ermöglichte, ihre Ergebnisse selbst besser zu reflektieren und einzuordnen bzw. die besondere Herausforderung zu verstehen.

An das Review schloss sich jeweils eine Retrospektive an, in der die Lernenden ihren Lernprozess, moderiert durch die methodische Begleiterin, reflektierten. Als Hilfsmittel wurde jeweils ein kleiner Fragebogen mit sechs kurzen Fragen eingesetzt. War der Fragebogen zunächst nur als Evaluationsinstrument für die begleitende Evaluation im Forschungsprojekt gedacht, zeigte sich rasch, dass er sich als Einstieg für die Diskussion zum Lernprozess gut eignete. Eine der Fragen richtete sich zum Beispiel auf das Verstehen der 


\section{Zielbild entwickeln}

Welche Vision von einer veränderten Information/ Kommunikation/ Zusammenarbeit verfolgt MARVECS mit dem Social Intranet?

Es gibt 2 prinzipiell zu unterscheidende Ansätze, um ein Zielbild zu entwerfen:

- Problemorientiert: eher auf Verbesserung/Optimierung ausgerichtet,

- Lösungsorientiert: eher auf Neuerung/Innovation ausgerichtet.

Macht euch schlau, was dahintersteckt und probiert selbst aus, was euch besser gefält bzw. was zum Vorhaben "Intranet" besser passt. Entscheidet euch für eine der Optionen und entwickelt euer "Zielbild Intranet".

Bereitet zudem den Test eures "Zielbilds Intranet" an der Geschäftsführerin und an einem/einer Betroffenen vor. Führt den Test durch.

Akzeptanzkriterien:

- Ihr könnt eure Entscheidung begründen.

- Das „Zielbild Intranet“ beschreibt aus Sicht MARVECS die zukünftige Situation und hebt deren Attraktivität (für die Intranetnutzer bzw. die Geschäftsführung) hervor.

- Das Zielbild nimmt keine technische Lösung vorweg.

- Die Test-Vorbereitung umfasst auch Überlegungen, wie das Feedback eingeholt werden soll und woran zu erkennen sein wird, ob euer Zielbild ein Erfolg ist.

Quellen:

- Buch, J. Little: Lean Change Management, S. $121 \mathrm{ff}$.

- Folie mit Comics zu Problem- vs. Lösungsorientiert

- Folie mit „Wunderfrage“

Abb. 9.2 Lernaufgabe „Zielbild entwickeln“

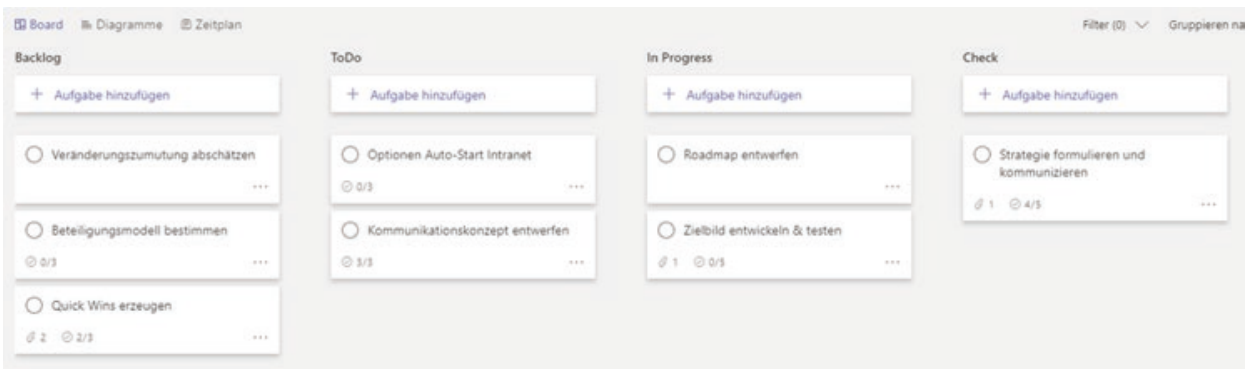

Abb. 9.3 Blick auf das Kanban-Board zur Visualisierung des Lernfortschritts

Lernaufgaben. Das gab den Lernenden gleich in der ersten Retrospektive die Chance, für die neuen Lernaufgaben eine klarere Aufgabenformulierung einzufordern.

In der Regel dauerte die Retrospektive nicht länger als zehn bis fünfzehn Minuten.

Im dritten und letzten Teil des Etappenwechsels wurden die Aufgaben für die nächste Etappe geplant: Welche Aufgabe wollten die Lernenden bearbeiten? Wie wollten sie dabei 
vorgehen? Zweimal wurden dabei auch Lernaufgaben angepasst, um den aktuellen Bedarf des Intranet-Projekts besser zu bedienen.

Auch dieser Teil nahm mit bis zu dreißig Minuten relativ viel Zeit in Anspruch: Hier galt es den Stand des Intranet-Projekts zu berücksichtigen und die nächste Etappe im Detail zu besprechen.

Dies sowie das ausführliche Review führten dazu, dass die Etappenwechsel zumeist fünfzehn Minuten länger dauerten als die ursprünglich eingeplanten 1,5 Stunden.

\section{Kick-out des Lernprojekts am Tag vor dem Release des Social Intranet}

Das letzte Meeting zum Kick-out fand einen Tag vor dem Release des Social Intranet statt. Im letzten Review wurden noch einmal die Ergebnisse der Lernaufgaben besprochen. Die meiste Zeit galt nun aber dem Feiern der Erfolge und einem letzten Erfahrungsaustausch. Dazu stieß die fachliche Begleiterin eine Diskussion darüber an, in welchen Punkten sich die Lernenden - als Leitende des Intranet-Projektes - gut vorbereitet fühlten und welche Herausforderungen sie noch sahen bzw. zu erwarten hatten.

Die letzte Retrospektive nutzte die methodische Begleiterin dazu, noch einmal den gesamten Lernprozess zu betrachten: Was war gut gelungen, was weniger? Welche Unterstützung wünschten sich die Lernenden im agilen Lernen vonseiten der methodischen Begleitung für zukünftige Lernprojekte?

\subsection{Beobachtungen und Lessons Learned}

Dieses Projekt ist ein gutes Beispiel dafür, wie an konkreten Projektherausforderungen gelernt werden kann und wie diese individuellen Erkenntnisse zu einer Entwicklung des Unternehmens beitragen können. Dies zeigen auch die Rückmeldungen der Beteiligten nach Abschluss des Projekts:

„Ohne die Aufgabe zu Beteiligungsmodellen wären wir nie auf die Idee gekommen, das Intranet viral und trotzdem gezielt gesteuert zur breiten Anwendung zu bringen." (Leiterin Finance \& Human Resources)

„Über die Optionen-Aufgabe wurde schnell klar, welche die einzig akzeptable Option für den Start der Intranetseite ist.“ (IT Operations Manager)

„Die Vorgehensweise mit regelmäßigen Tests in kurzen Abständen ermöglicht es, nachzujustieren und so schnell und agil auf Herausforderungen zu reagieren." (Leiterin Finance \& Human Resources)

\section{Lernziele und Motivation: Vom Skeptiker zum Überzeugungstäter}

Die Leiterin Finance \& Human Resources war von Anfang an vom großen persönlichen Nutzen des Lernprojekts überzeugt. Der IT Operations Manager hatte hier zu Anfang noch eher eine abwartende bis skeptische Haltung: War es für seine Aufgabe als IT-Experte wirklich hilfreich, eine Strategie, ein Zielbild o. ä. formulieren zu können? Das änderte 
sich spätestens zum Ende der dritten Etappe: Zu diesem Zeitpunkt konnte er mithilfe des Zielbilds und der Roadmap seinen Standpunkt darstellen und verteidigen. Zudem konnte er zum Beispiel anhand der Aufgabe „die beste Option finden“ einen Streitpunkt im Intranet-Projekt rasch auf fachlicher Ebene klären.

\section{Lernorganisation: Unrhythmisch auf der Überholspur unterwegs}

Die unrhythmisch angelegte Lernorganisation ohne zeitlich enge Abstimmung der Etappentreffen des Lernens auf die Treffen des Intranet-Projekts erwies sich im Nachhinein nicht als Nachteil. Die schlechtere Alternative wäre gewesen, die Etappentreffen, unterstützt mit Web-Conferencing-Tools, online durchzuführen, zumal der Austausch mit der fachlichen Begleiterin im persönlichen Gespräch in den Reviews als wertvoll wahrgenommen wurde. Dennoch ist eine Abstimmung von Change- und Lernprojekt aufeinander prinzipiell zu empfehlen, um noch gezielter das Change Projekt zu bedienen und unmittelbarer neu Gelerntes zur Anwendung zu bringen.

Gleiches gilt für den gegenüber dem Intranet-Projekt verzögerten Start des Lernprojekts: Zwar ist es gelungen, Versäumtes nachzuholen, allerdings schafft zum Beispiel ein Zielbild einen noch größeren Nutzen, wenn es bereits zum Start eines Change Vorhabens vorliegt.

\section{Die Lernbegleitung: Yin und Yang}

Von Vorteil war, dass die methodische Begleiterin des Intranet-Projekts auch die methodische Begleitung im Lernprojekt übernehmen konnte. Sie war über den Fortschritt, über Stimmungslagen oder Besonderheiten im Bilde. Auf der anderen Seite war es von großem Nutzen, dass die externe fachliche Begleiterin umfassenderes Erfahrungswissen einbringen und die Fortschritte sowie Ergebnisse mit Abstand betrachten konnte. Interne und externe Sichtweisen ergänzten sich hier optimal.

\section{Die Auftraggeberin: Nicht anwesend und doch präsent}

Die Geschäftsführerin als Auftraggeberin nahm an keinem der Meetings zum Etappenwechsel teil, auch nicht am Review, was zuallererst ihrem Terminkalender geschuldet war. Dies wurde bewusst in Kauf genommen, da sie über die bestehende Regelkommunikation mit der Leiterin Finance \& Human Resources (als einer der beiden Lernenden) gut informiert war. Zudem nahm sie an Tests zu Zwischenergebnissen der einzelnen Lernaufgaben teil (etwa zum Zielbild, s. Abb. 9.2).

Unabhängig davon war sicher die bei MARVECS gelebte Eigenverantwortungs- und Vertrauenskultur förderlich dafür, dass das selbstgesteuerte agile Lernen so gut gelingen konnte.

\section{Technische Unterstützung: Altbekanntes bewährt sich gut}

Zur Bereitstellung der Lernaufgaben, zur Unterstützung der Planung sowie zur Visualisierung des Lernfortschritts wurde das Planungstool MS Teams genutzt. Dies hat sich bewährt, zumal die Voraussetzungen optimal waren: Das Tool unterstützt alle wesentlichen 
Funktionen, alle Beteiligten im Lernprozess kannten das Tool aus der täglichen Arbeit und es wurde auch zur Organisation der Projektaufgaben im Intranet-Projekt genutzt.

\subsection{Was bleibt}

Mit dem Kick-out des Lernprojektes und dem Release des Social Intranet hat das Steuerungs- und Lernteam sein eigentliches Ziel noch nicht erreicht: Das Intranet lebt und erfüllt seinen Zweck erst dann, wenn die Kollegen das Intranet zum intensiven Austausch untereinander und in der täglichen Arbeit für den Zugriff auf digitale Anwendungen nutzen.

Für die Anlaufphase des Social Intranet nach dem Release haben sich die Leiterin Finance \& Human Resources sowie der IT Opreations Manager deshalb vorgenommen, gemeinsam die Roadmap und das Kommunikationskonzept fortzuschreiben sowie das Eintreten der erhofften Quick Wins wie geplant zu überprüfen.

Über das Intranet-Projekt hinaus brachten beide den Ansatz, ein Zielbild zu formulieren und im Steuerungsteam sowie mit der Geschäftsführerin abzustimmen, in einem neuen Projekt zur Anwendung. Die Leiterin Finance \& Human Resources agiert inzwischen als interne fachliche Beraterin für ein neues Change Vorhaben und gibt auf diesem Weg ihr neu erworbenes Wissen zu agilem (Lean) Change Management und zur Beteiligung von Betroffenen weiter.

Open Access Dieses Kapitel wird unter der Creative Commons Namensnennung - Weitergabe unter gleichen Bedingungen 4.0 International Lizenz (http://creativecommons.org/licenses/ by-sa/4.0/deed.de) veröffentlicht, welche die Nutzung, Vervielfältigung, Bearbeitung, Verbreitung und Wiedergabe in jeglichem Medium und Format erlaubt, sofern Sie den/die ursprünglichen Autor(en) und die Quelle ordnungsgemäß nennen, einen Link zur Creative Commons Lizenz beifügen und angeben, ob Änderungen vorgenommen wurden. Wenn Sie das Buch oder Teile daraus remixen, verändern oder anderweitig direkt darauf aufbauen, dürfen Sie Ihre Beiträge nur unter derselben Lizenz wie das Original verbreiten.

Die in diesem Kapitel enthaltenen Bilder und sonstiges Drittmaterial unterliegen ebenfalls der genannten Creative Commons Lizenz, sofern sich aus der Abbildungslegende nichts anderes ergibt. Sofern das betreffende Material nicht unter der genannten Creative Commons Lizenz steht und die betreffende Handlung nicht nach gesetzlichen Vorschriften erlaubt ist, ist für die oben aufgeführten Weiterverwendungen des Materials die Einwilligung des jeweiligen Rechteinhabers einzuholen.

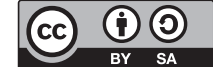

